\title{
Understanding the Real-World Performance of Carrier Sense
}

\author{
Kyle Jamieson, Bret Hull, Allen Miu, Hari Balakrishnan \\ MIT Computer Science and Artificial Intelligence Laboratory \\ The Stata Center, 32 Vassar St., Cambridge, MA 02139 \\ \{jamieson, bwhull, aklmiu, hari\}@csail.mit.edu
}

\begin{abstract}
Carrier sense is a fundamental part of most wireless networking stacks in wireless local area- and sensor networks. As increasing numbers of users and more demanding applications push wireless networks to their capacity limits, the efficacy of the carrier sense mechanism becomes a key factor in determining wireless network capacity.

We describe how carrier sense works, point out its limitations, and advocate an experimental approach to studying carrier sense. We describe our current testbed setup, and then present preliminary experimental results from both a 60-node sensor network deployment and a small-scale 802.11 deployment. Our preliminary results evaluate how well carrier sense works and expose its limitations.
\end{abstract}

\section{Categories and Subject Descriptors}

C.2.1 [Computer Systems Organization]: ComputerCommunication Networks-Network Architecture and Design

\section{General Terms}

Measurement, performance, design, experimentation.

\section{Keywords}

Carrier sense, medium access control

\section{INTRODUCTION}

Carrier sense is a mechanism common to almost all modern wireless communication stacks. Consequently, an understanding of the strengths and weaknesses of carrier sense in the real world has implications for many wireless systems.

The basic idea of carrier sense is as follows. Before transmitting, a sender listens to the channel and assesses whether a nearby node is transmitting. If no nearby node is transmitting, the sender transmits immediately. If a nearby node

Permission to make digital or hard copies of all or part of this work for personal or classroom use is granted without fee provided that copies are not made or distributed for profit or commercial advantage and that copies bear this notice and the full citation on the first page. To copy otherwise, to republish, to post on servers or to redistribute to lists, requires prior specific permission and/or a fee.

SIGCOMM'05 Workshops, August 22-26, 2005, Philadelphia, PA, USA.

Copyright 2005 ACM 1-59593-026-4/05/0008 ...\$5.00.

\begin{tabular}{lll}
\hline & \multicolumn{2}{c}{ Experimental testbed } \\
\cline { 2 - 3 } & Sensor network & $\mathbf{8 0 2 . 1 1}$ \\
\hline Radio & Chipcon CC1000 & Atheros 5212 \\
Data rate & 38.4 Kbps & 2 to 60 Mbps \\
Modulation & FM narrowband & $\begin{array}{l}\text { OFDM spread } \\
\text { spectrum }\end{array}$ \\
Nodes & 60 & 3 \\
\hline
\end{tabular}

Table 1: The two experimental testbeds used in this paper.

is transmitting, the sender defers, waiting for some time after the end of the intervening transmission. Then the sender repeats the same carrier sense-defer process.

Carrier sense is a part of the medium access control (MAC) layer of the radio stack. Well-informed MAC decisions are crucial to maximizing the capacity of a broadcast radio medium. Failed transmissions not only waste energy, but also have the potential to corrupt other transmissions in the network, reducing aggregate capacity. Deferring a transmission has the potential of wasting a good transmission opportunity, thereby reducing capacity.

Carrier sense is at best a heuristic to avoid these problems, because the sender and receiver are in different locations, and the sender makes the carrier sense decision based on local information. Determining how well and why carrier sense works in real wireless networks should be the focus of experimental study.

Experimental studies of carrier sense are hard for two reasons. First, it is difficult to design an experiment to separate interference due to a small number of nearby radios from interference due to a large number of far-off radios. Second, it is dangerous to generalize conclusions from one particular experimental setup.

We are building two testbeds to study carrier sense. The first is a 60-node wireless sensor network communicating with narrowband FM radios. The second will be a mediumscale 802.11 testbed communicating with 802.11 spread spectrum OFDM radios. Table 1 summarizes the current state of our experimental testbeds.

Our preliminary experimental results show that while carrier sense improves link qualities at all traffic loads, it leaves room for performance improvements. Furthermore, carrier sense can actually reduce capacity under extreme loads. 


\section{RELATED WORK}

There is a large body of theoretical work on carrier sensing that has not yet been experimentally evaluated. The following are some examples.

As part of the MACA [4] MAC protocol for wireless packet radio, Karn proposes "throwing out" the carrier sense line on packet radio transceivers, and instead relying on an RTS/CTS exchange to protect against interfering transmissions. While the idea of RTS/CTS has remained popular in wireless localarea networks and sensor networks, the idea of discarding carrier sense has not been evaluated experimentally.

More recently, Yang and Vaidya [11] examine the choice of the carrier sense range and its effect on capacity, taking into account MAC layer overhead. We share their observations about the impact that carrier sense and varying traffic loads have on overall network capacity. We propose to experimentally evaluate the simulation results in their work at both large and small scales.

Fuemmeler et al. [3] study the choice of carrier sense threshold (defined in Section 3.1) and transmit power for 802.11 mesh networks. They conclude that senders should keep the product of their transmit power and carrier sense threshold equal to a fixed constant.

Zhu et al. present an analytical model for deriving an optimal carrier sense threshold [12], but their model does not take into account MAC layer overhead. They have also proposed a distributed algorithm that adapts the carrier sense threshold of an 802.11 mesh network [13], and presented simulation results validating its efficacy.

Desilva et al. [2] found that carrier sense can unnecessarily suppress an 802.11 receiver from responding to RTS messages. They observe that a successful reception of a RTS message is a good indication that subsequent transmissions from the RTS sender can overcome the current noise levels observed at the receiver, even when the noise level is within carrier sensing range. To increase efficiency, they propose 802.11 receivers use a different threshold for carrier sense prior to transmitting a CTS message.

\subsection{Experimental work}

In a small-scale 802.11 testbed, Li et al. observe multihop interference which limits the end-to-end throughput a short forwarding chain of 802.11 nodes can achieve [5]. They note that because the interference range of the radio is greater than the carrier sensing range, carrier sense alone does not solve the problem.

As part of CODA [8], Wan et al. propose a method for detecting congestion in a wireless sensor network called channel sampling. When a packet is waiting to be sent, the sensor samples the state of the channel at a fixed interval. Based on the number of times the channel is busy, it calculates a utilization factor. The node infers congestion if utilization rises above a certain level. We speculate that congestion control can improve network capacity when carrier sense fails.

Whitehouse et al. present a collision detection and recovery technique for wireless networks that takes advantage of the capture effect [10]. Their technique detects packet collisions by searching for a preamble sequence throughout packet reception and recovers by re-synchronizing to the last detected preamble. We note that capture is a phenomenon that few protocols make explicit use of and that it might be exploited to make a more informed channel access decision.

\section{CARRIER SENSE}

In this section we describe several carrier sense mechanisms. In addition, we offer reasons for why carrier sense may sometimes make incorrect channel access decisions.

\subsection{Design}

Common to almost all carrier sense implementations is an automatic gain control (AGC) system. The baseband processor (or its software equivalent) monitors the percentage of time that the A/D converters in the baseband processor are saturated, and adjusts the $\mathrm{A} / \mathrm{D}$ gain accordingly. Instantaneous signal strength $(\rho)$ is a function of the AGC gain. It represents the amount of energy being received at the antenna at any instant in time.

We now list common ways of detecting an incoming transmission:

1. Preamble detection is the simplest of the available methods. Each transmission begins with a unique preamble sequence, so it is highly likely that there is an incoming transmission if the radio decodes a preamble.

2. The $A G C$ unlock indicator becomes true when the A/D converters' outputs fluctuate outside of a predefined lock window.

3. The Energy detect (ED) indicator is a carrier sense mechanism common to many extant radios. It is based on signal strength readings obtained from the radio front end. Over the periods that there are no incoming transmissions, senders time-average instantaneous signal strength readings into a quantity called squelch $(\sigma)$. The squelch represents the "noise floor" of the network: the signal strength of background noise. Just before a transmission, the sender makes its carrier sense decision with a comparison between $\rho$ and $\sigma$. If $\rho>\sigma$, then carrier sense is busy. Otherwise, carrier sense is idle and the sender may begin transmission. Alternately, some radios use a fixed threshold for $\sigma$, commonly referred to as the carrier sense threshold.

4. The Decorrelation amplitude is an indicator unique to spread-spectrum radios. It measures how well an incoming signal correlates with the known pseudo-random noise code of the spread-spectrum radio. If it is greater than a fixed threshold, then the decorrelation amplitude indicator is true.

\subsection{Room for improvement?}

Carrier sense is not always a good predictor of transmission success because it relies on channel measurements at the sender to infer the probability of reception at the receiver. However in many cases, no correlation exists between channel conditions at the sender and at the receiver. This lack of correlation is often due to exposed terminals, the aggregate effect of distant nodes raising the noise floor, and capture.

On a local level, carrier sense may perform poorly when exposed terminals are present. Consider the following situation. Two transmitters, nodes $A$ and $B$, are both within radio range of each other. The intended recipients of their transmissions, nodes $A^{\prime}$ and $B^{\prime}$ respectively, are each within range of only one transmitter, and hence, could each simultaneously receive a packet from the intended transmitter. 
Unfortunately, carrier sense would only allow one transmission to take place. Whichever node lost the CSMA contention period would sense a busy channel and wait for the other node's transmission to complete.

Additionally, carrier sense may be a poor predictor of transmission success if interference comes from a large number of distant nodes rather than a few local neighbors. When interference is local and nodes are within each other's transmission range, carrier sense or an RTS/CTS exchange may be a good method of contending for the channel. However, because a node's interference range is much larger than its transmission range, distant transmitters can easily impact local transmissions. In aggregate, these distant transmitters raise the overall "noise floor" of the network, reducing link quality. Carrier sense as described in Section 3.1 cannot mitigate this type of interference.

Finally, carrier sense may be overly conservative in the presence of capture, a phenomenon in which a radio successfully demodulates one of multiple overlapping transmissions of the same frequency. Specifically, this property means that concurrent transmissions are possible by a set of nodes well within each others' transmission range. This differs from the carrier sense assumption that only one node should be transmitting in the receiver's radio neighborhood.

\section{EXPERIMENTAL TESTBEDS}

In this section we describe the details and current status of our experimental testbeds, and present preliminary results evaluating the efficacy of carrier sense.

\subsection{Sensor network experimental setup}

The first experimental setup is a 60-node indoor wireless sensor network testbed. Each node is a Crossbow Mica2, which has an Atmel ATmega128L microcontroller with $4 \mathrm{~KB}$ of RAM, $128 \mathrm{~KB}$ of flash, and a CC1000 radio. The radio operates at $433 \mathrm{MHz}$, transmits at $38.4 \mathrm{Kbps}$, and uses frequency modulation. Each node is attached to a Crossbow MIB600 interface board that provides both power and an Ethernet backchannel for programming and data collection. We have deployed nodes over an area of 16,076 square feet on one floor of our office building, with liberal coverage throughout the floor and a higher than average density in one corner of the floor. We use Motelab [9] to manage the testbed.

Our sensor network nodes run a variant of B-MAC [7] on the Chipcon CC1000 [1] radio. B-MAC uses energy detect (see Section 3.1) to sense carrier. In our sensor network experiments, every node transmits broadcast data at the nominal rate to any nodes that can hear it. Nodes do not forward any data, so the communication pattern is local throughout the network.

\subsection{11 experimental setup}

The second experimental setup is a small testbed consisting of three indoor 802.11 nodes in close proximity. Two nodes act as senders and are placed at about six meters apart. The receiver is placed approximately 12 meters from each of the senders. Each node is equipped with an Atheros $802.11 \mathrm{a} / \mathrm{b} / \mathrm{g}$ combo card driven by the madwifi driver [6].

802.11 chipsets such as the one in our testbed use a combination of the mechanisms listed in Section 3.1 to detect an ongoing transmission. The carrier sense mechanism takes all three indicators into account when making a carrier sense
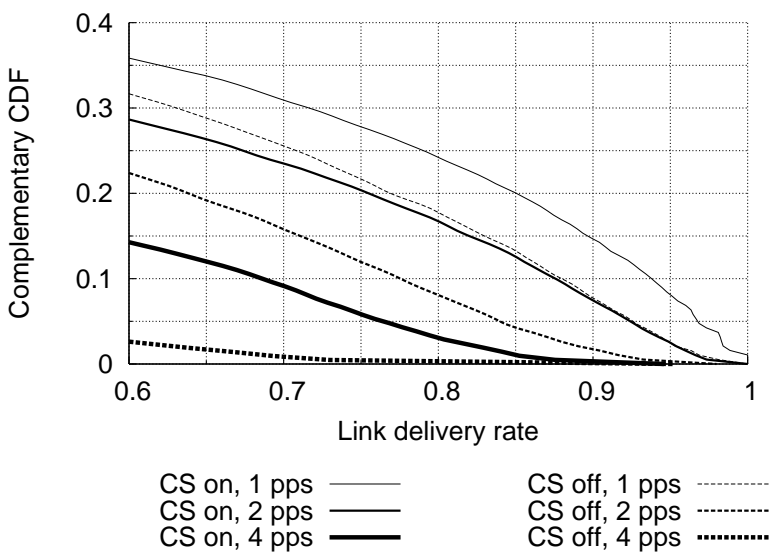

Figure 1: The complementary cumulative fraction of node pairs that achieve link delivery rates greater than 0.6 in the sensor network testbed. "CS on (off)" means that sensors perform (do not perform) carrier sense as described in Section 3.1.

decision. Typically, wireless manufacturers determine how to logically combine the indicators to yield the carrier sense output.

\subsection{Carrier sense improves link delivery rates}

As mentioned previously, carrier sense may be a poor predictor of transmission success in a large network where interference can come from a large number of distant nodes. The purpose of this experiment is to evaluate the efficacy of carrier sense in a large, dense sensor network.

Figure 1 shows the distribution of link delivery rates across links that are most likely to be useful to higher-level protocols (greater than $60 \%$ link delivery rate). The same trends shown in the figure hold for the bottom $60 \%$ links. Figure 1 shows that the probability of a single transmission succeeding increases when sensors perform carrier sense. As the amount of traffic in the network increases, link quality decreases sharply. Furthermore, the more traffic in the network, the more carrier sense improves link quality. Carrier sense improves link quality by a small amount when each node offers 1 pps, but by a significant amount when each node offers 4 pps. At low loads, the channel is idle most of the time, and we hypothesize that therefore most losses are path losses, which carrier sense cannot prevent. At high loads, the channel is busy most of the time, and therefore carrier sense sometimes helps avoid collisions.

\subsection{Limitations of carrier sense}

We now establish, in our 802.11 testbed, at which bit rates carrier sense is ineffective due to the capture effect described above in Section 3.2. We discover that carrier sense can be ineffective at low data rates when the capture effect is most prevalent. Consequently, the standard carrier sense algorithm can lead to many mispredictions and wasted transmission opportunities in practice.

We place two 802.11 senders $(A$ and $B)$ in close proximity such that they are well within communication range of each other at all bit rates. Because the typical carrier sense range is greater than communication range, the two senders are also well within carrier-sensing range of each 


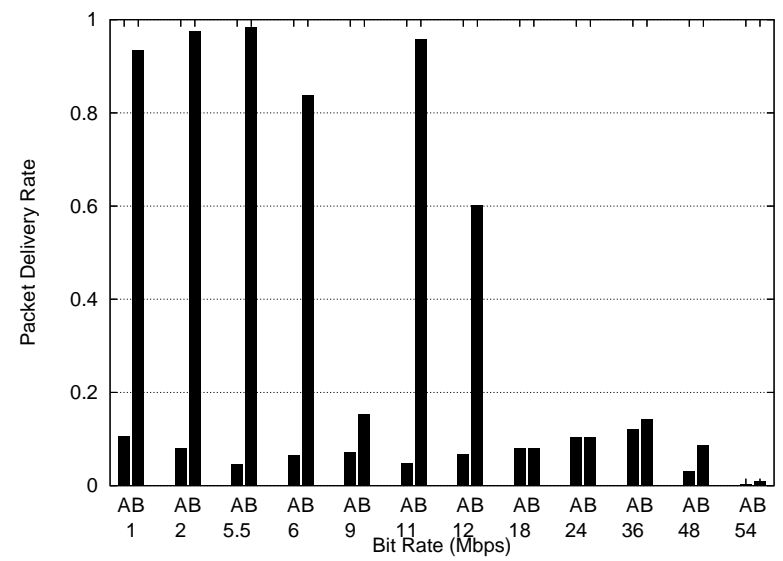

Figure 2: Packet delivery rate in the 802.11 network for two simultaneous senders, each transmitting at the saturation rate at the selected bit rate. Carrier sense is effectively disabled in this experiment.

other. We modified the driver to disable randomized backoff, which gives the effect of disabling carrier sense and allows two senders to transmit packets simultaneously.

The results in Figure 2 show low packet delivery rates at high bit rates. Due to mutual interference from the simultaneous transmissions, the receiver fails to decode most data frames transmitted by either sender. As bit rate decreases, however, we observe that the receiver often captures one of the senders $(B)$, thus achieving a very high frame delivery rate from $B$. The degree of capture is surprisingly large at low bit rates: the link between sender $B$ and the receiver achieves a delivery rate of over $80 \%$ for bit rates $1,2,5.5$, and $6 \mathrm{Mbps}$.

The capture effect is attributed to the relative difference of the received signal strength between the two senders' transmitted frames. In our experiments, the average RSSIs of $A$ and $B$ are $-57.2 \mathrm{dBm}$ and $-48.8 \mathrm{dBm}$ respectively. In general, the probability of capture increases as the ratio between the RSSI of the captured signal and the RSSI of the drowned-out signal increases. Also, the minimum required signal ratio for capture to take place decreases as bit rate decreases.

Current carrier sense schemes are oblivious to the capture effect. Consequently, it mispredicts transmission failures and wastes potential transmission opportunities when they exist. In our experiments, if randomized backoff were enabled (thereby, allowing carrier sense to take full effect) and we assume that the intended destination of $A$ and $B$ 's transmissions are different, then sender $B$ would have deferred its transmissions due to sender $A$ 's transmissions, even though the receiver could capture $B$ 's transmissions at low bit rates.

We can improve network efficiency by designing a carrier sense mechanism such that it is capture-aware: it should make transmission deferral decisions based on the bit rates being used and the received signal strength ratios observed at all of the nearby receivers. For example, if $A$ 's intended receiver can capture $B$ 's transmissions, carrier sense should be used to defer $B$ 's transmission to prevent it from interfering with $A$ 's transmission. On the other hand, if $A$ 's intended receiver can tolerate a parallel transmission from $B$ without significantly affecting $A$ 's delivery rate, carrier sense should be suppressed to make efficient use of the available transmission opportunities.

\subsubsection{How often do carrier-sensing senders miss trans- mission opportunities?}

Recall that just before sending a packet, transmitters using the energy detect method of carrier sense (described fully in Section 3.1) compare their current squelch $\sigma$ with the instantaneous signal strength $\rho$. To evaluate how well energy detect works, we now examine how well the MAC performs as a function of $\rho$ and $\sigma$.

Suppose a sender carrier-senses the channel busy, but had the sender transmitted anyway, the packet would have been successfully received. We call such an event a false-positive carrier sense. Figure 3 quantifies how often this happens, parameterized on the actual carrier sense reading at the sender. Each sender in this experiment uses the energy detect mechanism to sense carrier before transmitting. In Figure 3 (left), senders wait until $\rho<\sigma$ before transmitting. In Figure 3 (right), senders record $\rho$ and $\sigma$, but do not wait for $\rho$ to fall below $\sigma$ before transmitting. The figures show average link delivery rates as a function of $\rho$ and $\sigma$. The set of links we consider are those with an overall loss rate of less than $20 \%$. These links are of most use to routing and higher layers.

First, note that carrier-sensing senders make no transmissions above the diagonal line $\rho=\sigma$ in Figure 3 (left). Looking at Figure 3 (right), we see that senders that ignore carrier sense achieve rather high link qualities above the diagonal $\rho=\sigma$. This suggests that the energy detect method of carrier sense may be forgoing some good transmission opportunities.

\subsection{When to abandon carrier sense}

Figure 4 shows the distribution of achieved throughput over all links in the sensor network. At 1 and $4 \mathrm{pps}$, enabling per-packet carrier sense results in greater throughput than disabling it altogether. This is because while per-packet carrier sense may slow down an individual transmission, our experiments take this into account and transmit such that the nominal transmission rate is equal to the actual transmission rate. At 8 pps, however, some carrier-sensing nodes cannot keep up with the offered load, because it takes too much time to carrier sense. As a result, throughput suffers and consequently, nodes achieve higher throughput with carrier sense disabled. Thus even though carrier sense improves link quality at high loads, under extremely high loads, the improvement in link quality might not be worth the time it takes to carrier sense.

\section{CONCLUSION}

We have argued for an experimental approach to the study of carrier sense in radio networks. With the goal of maximizing network capacity in mind, we have highlighted the existing problems of carrier sense, and presented experimental results about its efficacy in two separate network testbeds. The first is a large-scale deployment of narrowband FM radio sensor nodes, and the second is a small-scale deployment of wideband 802.11 radios.

In the future we plan to grow our 802.11 testbed so that we can study the effects of global interference in a spreadspectrum system. Additionally, we intend to build a parallel sensor testbed using 802.15.4 radios to confirm the generality of our findings across different radio technologies. Using 

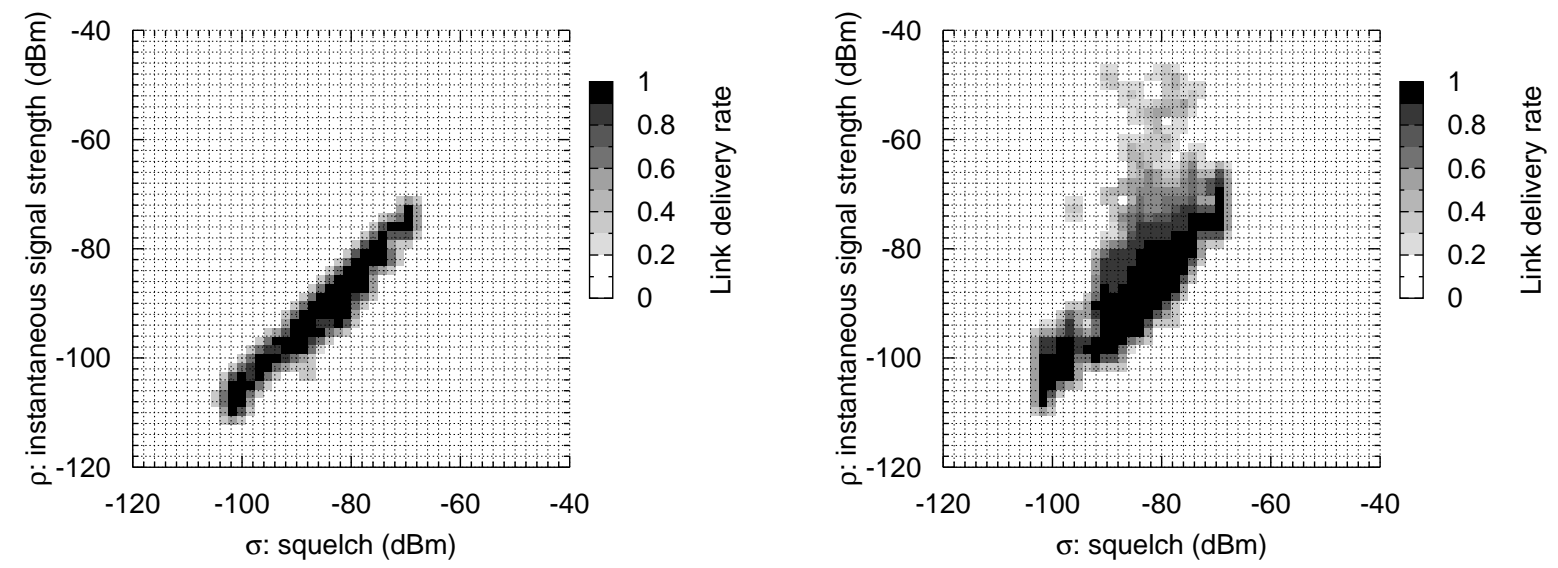

Figure 3: Average packet delivery rate in the sensor network testbed as a function of squelch and instantaneous signal strength just prior to transmission. All nodes send data at a rate of 4 pps/node. We measure both independent variables at the sender, with carrier sense enabled (left) and disabled (right).
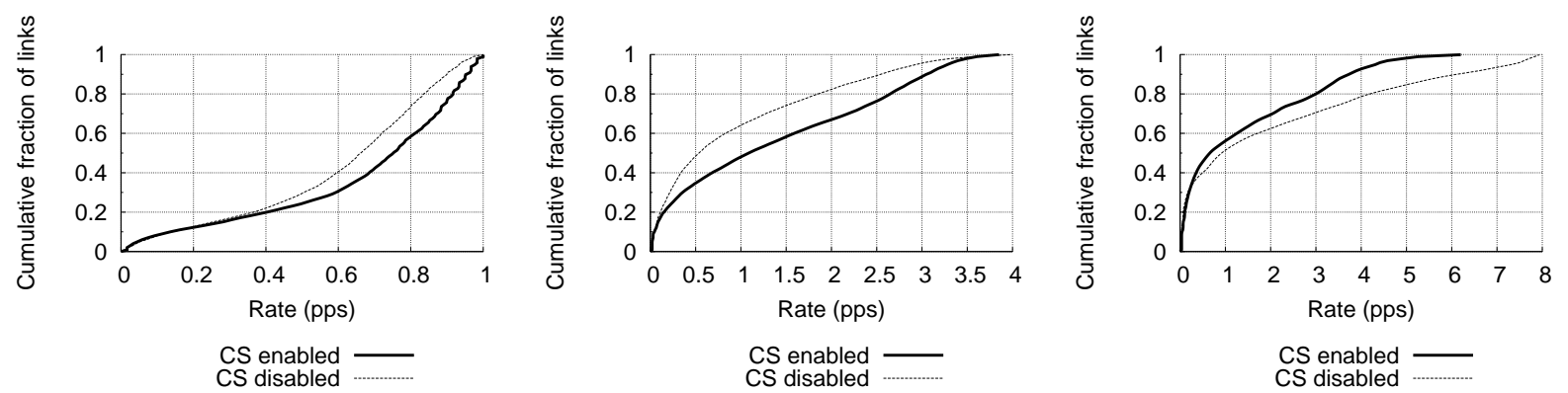

Figure 4: The achieved link throughput distribution in the sensor network when each node offers 1 pps (left). Duplicate experiments at an offered load of 4 pps (center) and 8 pps (right). Under extreme load, disabling carrier sense improves throughput.

the results of our performance study, we plan to design and implement a system to increase network capacity by modifying the carrier sense decision process. The measurements we gather from the testbeds presented in this paper will guide the formulation of such an algorithm.

\section{Acknowledgments}

Thanks to Srikanth Kandula for his helpful feedback.

\section{REFERENCES}

[1] Chipcon Corporation. CC1000 Transceiver Datasheet. http://www.chipcon.com.

[2] Desilva, S., And Boppana, R. On the Impact of Noise Sensitivity on Performance in 802.11 Based Ad Hoc Networks. In Proc. of the IEEE International Conf. on Communication (Paris, France, June 2004).

[3] Fuemmeler, J., Vaidya, N., and Veeravalli, V. Selecting Transmit Powers and Carrier Sense Thresholds for CSMA Protocols. Tech. rep., University of Illinois at Urbana-Champaign, October 2004.
[4] KARN, P. MACA-A New Channel Access Method for Packet Radio. In Proc. of the 9th ARRL Computer Networking Conf. (London, Ontario, 1990).

[5] Li, J., Blake, C., Couto, D. S. J. D., Lee, H. I., AND Morris, R. Capacity of Ad Hoc Wireless Networks. In Proc. of the ACM MobiCom Conf. (Rome, Italy, July 2001), pp. 61-69.

[6] Madwifi: Multiband Atheros Driver for WiFi. http://madwifi.sourceforge.net/.

[7] Polastre, J., Hill, J., And Culler, D. Versatile Low-Power Media Access for Wireless Sensor Networks. In Proc. of the ACM SenSys Conf. (Baltimore, MD, November 2004), pp. 95-107.

[8] Wan, C.-Y., Eisenmen, S., And Campbell, A. CODA: Congestion Control in Sensor Networks. In Proc. of the ACM SenSys Conf. (Los Angeles, CA, November 2003), pp. 266-279.

[9] Welsh, M. And Werner-Allen, G. Motelab sensor network testbed software. http://motelab.eecs.harvard.edu.

[10] Whitehouse, K., Woo, A., Jian, F., Polastre, J., AND Culler, D. Exploiting The Capture Effect For 
Collision Detection and Recovery. In Proc. of the IEEE Em-NetS-II Workshop (May 2005).

[11] YAng, X., AND VAIDYA, N. On Physical Carrier Sensing in Wireless Ad Hoc Networks. In Proc. of the IEEE INFOCOM Conf. (Miami, FL, March 2005).

[12] Zhu, J., Guo, X., YAng, L. L., And Conner, W. S. Leveraging Spatial Reuse in 802.11 Mesh Networks with Enhanced Physical Carrier Sensing. In Proc. of the IEEE Intl. Conf. on Communications (Paris, France, June 2004), vol. 7, pp. 4004-4011.

[13] Zhu, J., Guo, X., Yang, L. L., Conner, W. S., Roy, S., And Hazra, M. M. Adapting Physical Carrier Sensing to Maximize Spatial Reuse in 802.11 Mesh Networks. Wiley Journal of Wireless Communications and Mobile Computing 4, 8 (December 2004), 933-946. 